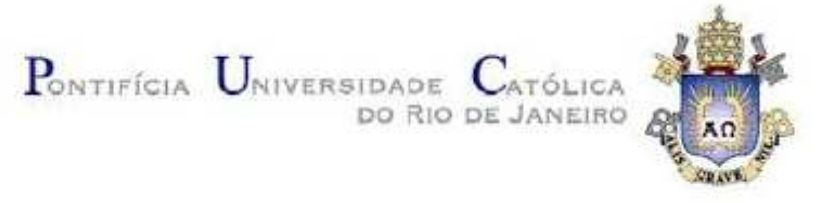

Ricardo de Menezes Barboza

Os Efeitos da Incerteza sobre Atividade
e Política Monetária no Brasil

Dissertação de Mestrado

Dissertação apresentada como requisito parcial para obtenção de grau de Mestre pelo Programa de PósGraduação em Macroeconomia e Finanças do Departamento de Economia do Centro de Ciências Sociais da PUC-Rio.

Orientador: Prof. Eduardo Zilberman

Rio de Janeiro

Fevereiro de 2017 


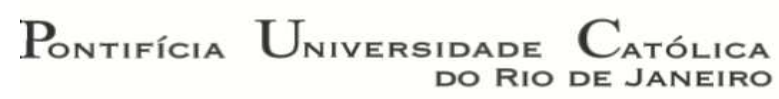

Ricardo de Menezes Barboza

\section{Os Efeitos da Incerteza sobre Atividade \\ e Política Monetária no Brasil}

Dissertação apresentada como requisito parcial para obtenção de grau de Mestre pelo Programa de PósGraduação em Macroeconomia e Finanças do Departamento de Economia do Centro de Ciências Sociais da PUC-Rio. Aprovado pela Comissão Examinadora abaixo assinada.

Prof. Eduardo Zilberman

Orientador

Departamento de Economia - PUC-Rio

Prof. Marcelo Cunha Medeiros Departamento de Economia - PUC-Rio

Prof ${ }^{a}$. Silvia Maria Matos EPGE/FGV

Profa. Mônica Herz Coordenadora do Centro de Ciências Sociais - PUC-Rio

Rio de Janeiro, 10 de fevereiro de 2017 
Todos os direitos reservados. É proibida a reprodução total ou parcial do trabalho sem a autorização da universidade, do autor e do orientador.

\section{Ricardo de Menezes Barboza}

Graduou-se em Economia pela Universidade Federal do Rio de Janeiro (UFRJ) em 2009. Fez Mestrado em Economia pela Universidade Federal do Rio de Janeiro (UFRJ) em 2012.

Ficha Catalográfica

Barboza, Ricardo de Menezes

Os efeitos da incerteza sobre atividade e política monetária no Brasil / Ricardo de Menezes Barboza ; orientador: Eduardo Zilberman. - 2017.

50 f. : il. color. ; $30 \mathrm{~cm}$

Dissertação (mestrado)-Pontifícia Universidade Católica do Rio de Janeiro, Departamento de Economia, 2017. Inclui bibliografia

1. Economia - Teses. 2. Incerteza. 3. Incerteza econômica. 4. Efeitos da incerteza. 5. Atividade econômica. 6. Política monetária. I. Zilberman, Eduardo. II. Pontifícia Universidade Católica do Rio de Janeiro. Departamento de Economia. III. Título. 


\section{Agradecimentos}

Ao Eduardo Zilberman, pela excelente orientação e pela confiança.

A Marcelo Medeiros e Silvia Matos, que me ajudaram a aprimorar o trabalho com comentários e sugestões. Ao Marcelo, em especial, agradeço pelo aprendizado transmitido nas diversas matérias e também pela amizade.

Aos professores Márcio Garcia, Tiago Berriel, Marco Cavalcanti, Carlos Viana, Diogo Guillén e Leonardo Rezende, pelos excelentes cursos ministrados.

Aos meus amigos Conrado Costa e Gabriel Vasconcelos, pela ajuda fundamental em diversos momentos críticos deste trabalho.

Ao Fabio Giambiagi, por me incentivar a fazer um segundo mestrado (inclusive me dando carta de recomendação) e pelo apoio diário, contribuindo decisivamente para meu desenvolvimento como economista.

Aos meus eternos mestres do Grupo de Conjuntura Econômica da UFRJ, Antônio Licha, Caio Prates da Silveira, Francisco Eduardo Pires de Souza, Margarida Gutierrez e Viviane Luporini, pela troca constante e saudável de ideias.

Aos meus amigos do Mestrado, Maurício Furtado, Gustavo Lima e Conrado Garcia, com quem tive (e espero continuar a ter) a oportunidade de conviver e aprender, tanto dentro quanto fora da sala de aula.

Aos meus amigos Daniel Brum, Rafael Feler, Guilherme Branquinho, Guilherme Tinoco, Gilberto Borça Jr, João Marco Braga, Luciano Machado, Marcio Gold Firmo, Fabio Roitman, André Sant'anna, Tiago Toledo, Sandro Garcia, Gustavo Rechdan, Rodrigo Madeira, Daniel Grimaldi, Breno Albuquerque e a todos os participantes do GINA, grupo de amigos economistas do BNDES.

À minha avó e ao meu avô (in memoriam), com quem desde cedo aprendi muito e que são meus referenciais de caráter e solidariedade.

Aos meus pais, Cintia, João e Aparecida, que sempre me ajudaram desde os primeiros passos. Não sei o que seria sem vocês, obrigado.

À Andrea, minha mulher e meu amor, que dividiu cada angústia desta trajetória ao meu lado, sempre me estimulando a seguir adiante, mesmo quando não havia qualquer luz no fim do túnel. Só o amor verdadeiro é capaz de fazer isso. 


\section{Resumo}

Barboza, Ricardo de Menezes; Zilberman, Eduardo. Os Efeitos da Incerteza sobre Atividade e Política Monetária no Brasil. Rio de Janeiro, 2017. 50p. Dissertação de Mestrado Profissional - Departamento de Economia, Pontifícia Universidade Católica do Rio de Janeiro.

Este trabalho tem um duplo objetivo. Em primeiro lugar, investiga qual o efeito da incerteza sobre a atividade econômica no Brasil. Para isso, são construídas diversas proxies que buscam captar o nível de incerteza vigente no Brasil (incerteza doméstica) e em vários de seus principais parceiros comerciais (incerteza externa). Em seguida, são estimados modelos de vetores autorregressivos (SVAR), em linha com Baker, Bloom e Davis (2016). Os resultados obtidos sugerem que a incerteza tem efeitos contracionistas relevantes sobre a economia brasileira. Em segundo lugar, estuda qual o efeito da incerteza sobre o poder da política monetária no Brasil. Para tanto, são construídos diversos modelos de vetores autorregressivos interativos (IVAR), tal como proposto por Aastveit, Natvik e Sola (2013), porém estimados por LASSO Adaptativo. As estimativas obtidas não corroboram a hipótese de que sob alta incerteza os efeitos da política monetária sobre a atividade são menores do que sob baixa incerteza. Este resultado, no entanto, não é robusto.

\section{Palavras-chave}

Incerteza; Incerteza econômica; Efeitos da incerteza; Atividade econômica; Política monetária. 


\section{Abstract}

Barboza, Ricardo de Menezes; Zilberman, Eduardo (Advisor). The Effects of Uncertainty on Activity and Monetary Policy in Brazil. Rio de Janeiro, 2017. 50p. Dissertação de Mestrado, departamento de Economia, Pontifícia Universidade Católica do Rio de Janeiro.

This work has a dual purpose. First of all, we investigate the effect of uncertainty on economic activity in Brazil. In order to do that, we construct several proxies which seek to capture the uncertainty level prevailing in Brazil (domestic uncertainty) and in several of our major trading partners (external uncertainty). Next, we estimate vector autoregressive (SVAR) models, in line with Baker, Bloom and Davis (2016). The results suggest that uncertainty has, in fact, contractionary effects on the activity in Brazil. Second, we study the effect of uncertainty on effectiveness of monetary policy in Brazil. Thus, we make use of interacted vector autoregressive (IVAR) models, as proposed by Aastveit, Natvik and Sola (2013), estimated, however, by Adaptive LASSO. Our estimates do not corroborate the hypothesis that under high uncertainty the effects of monetary policy on the activity are lower than under low uncertainty.

\section{Keywords}

Uncertainty; Economic Uncertainty; Uncertainty Effects; Economic Activity; Monetary Policy. 


\section{Sumário}

1 Introdução 12

2 Motivação Teórica 16

2.1. Um Modelo Teórico 16

3 Incerteza e Atividade Econômica no Brasil 19

3.1. Dados 19

3.2. Modelo SVAR 21

3.3. Resultados 23

3.4. Robustez 26

4 Incerteza e Efetividade da Política Monetária no Brasil 28

4.1. Modelo IVAR 28

4.2. Resultados 31

$\begin{array}{ll}\text { 4.3. Robustez } & 35\end{array}$

5 Conclusão 36

$\begin{array}{ll}6 & \text { Referências bibliográficas } \\ \end{array}$

7 Apêndice 41

7.1. Incerteza Doméstica 41

7.2. Incerteza Parceiros Comerciais $\quad 41$

7.3. Séries Modelo Básico 42

7.4. Testes de Raiz Unitária e Cointegração 43

7.5. Testes de Robustez 44

7.6. FRIs do IVAR $\quad 47$

7.7. Seleção de Variáveis do AdaLASSO 49 


\section{Lista de tabelas}

Tabela 1 - Correlação entre medidas de incerteza e atividade

Tabela 2 - Testes ADF e PP de Raiz Unitária

Tabela 3 - Testes de Cointegração de Johansen

Tabela 4 - Número de Variáveis Selecionadas pelo

AdaLASSO modelos com produção industrial

Tabela 5 - Número de Variáveis Selecionadas pelo

AdaLASSO nos modelos com IBC-Br 


\section{Lista de figuras}

Figura 1 - Indicador de Incerteza Econômica para o Brasil

Figura 2 - Resposta Indústria a choques de incerteza doméstica

Figura 3 - Resposta IBC-Br a choques de incerteza doméstica

Figura 4 - Resposta Investimento à incerteza doméstica

Figura 5 - Resposta das Medidas de Atividade aos Choques de Incerteza Externa

Figura 6 - Resposta Produção Industrial ao Choque Monetário sob alta e baixa incerteza

Figura 7 - Resposta IBC-Br ao Choque Monetário sob alta e baixa incerteza

Figura 8 - Resposta Produção Industrial e do IBC-Br diante de um choque monetário sob alta e baixa incerteza externa

Figura 9 - Séries normalizadas de incerteza doméstica

Figura 10 - Séries normalizadas de incerteza dos principais parceiros comerciais do Brasil

Figura 11 - Séries normalizadas dos modelos SVAR

Figura 12 - Respostas Indústria à incerteza doméstica

Figura 13 - Respostas IBC-Br à incerteza doméstica

Figura 14 - Respostas Investimento à incerteza doméstica

Figura 15 - Respostas Indústria à incerteza externa

Figura 16 - Respostas IBC-Br à incerteza externa

Figura 17 - FRIs do IVAR com os intervalos de confiança nos modelos com Produção Industrial 


\section{Lista de Abreviaturas}

\begin{tabular}{|c|c|}
\hline BCB & Banco Central do Brasil \\
\hline COPOM & Comitê de Política Monetária \\
\hline IBGE & Instituto Brasileiro de Geografia e Estatística \\
\hline IBRE & Instituto Brasileiro de Economia \\
\hline FGV & Fundação Getúlio Vargas \\
\hline IPEA & Instituto de Pesquisa Econômica Aplicada \\
\hline EUA & Estados Unidos da América \\
\hline PIM & Pesquisa Industrial Mensal \\
\hline Funcex & Fundação Centro de Estudos de Comércio Exterior \\
\hline CPB & Netherland Bureau of Economic Policy Analysis \\
\hline LASSO & Least Absolute Shrinkage and Selection Operator \\
\hline SVAR & Structural Vector Auto Regression \\
\hline IVAR & Interacted Vector Auto Regression \\
\hline Ibovespa & Índice da Bolsa de Valores de São Paulo \\
\hline PME & Pesquisa Mensal do Emprego \\
\hline EPU & Economic Policy Uncertainty \\
\hline $\mathrm{IBC}-\mathrm{Br}$ & Índice de Atividade Econômica do Banco Central \\
\hline OLS & Ordinary Least Squares \\
\hline SELIC & Sistema Especial de Liquidação e Custódia \\
\hline CUT & Custo Unitário do Trabalho \\
\hline
\end{tabular}


"Uncertainty is largely behind the dramatic collapse in demand. Given the uncertainty, why build a new plant, or introduce a new product now? Better pause until the smoke clears."

(Olivier Blanchard, 2009).

"Volatility has been over five times as high over the past six months as it was in the first half of 2007. The resulting uncertainty has almost surely contributed to a decline in spending."

(Christina Romer, 2009).

"Unresolved uncertainty can be a major inhibitor of investment. If energy prices will trend higher, you invest one way; if energy prices will be lower, you invest a different way. But if you don't know what prices will do, often you do not invest at all."

(Larry Summers, 2009). 